\title{
Keine Grenzen
}

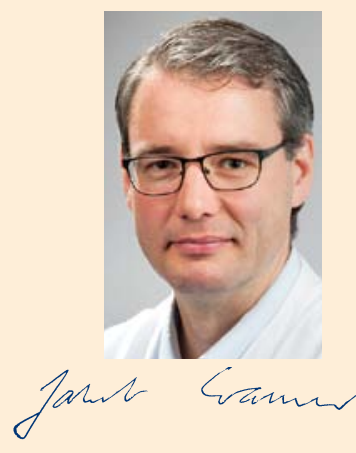

Liebe Kolleginnen und Kollegen,

am 14. und 15. März fand in Düsseldorf die 103. Jahrestagung der Deutschen Gesellschaft für Tropenmedizin und Internationale Gesundheit (DTG) statt, auf der auch ein neuer Vorstand gewählt worden ist - es wird ab Seite 90 ausführlich berichtet. Nachwuchsförderung, tropenmedizinische Aus-/Weiterbildung und die thematische Diversifizierung des Fachgebiets sind nur einige der wichtigen Themenfelder, die in den kommenden Jahren keineswegs an Bedeutung verlieren werden.

Auf der Tagung wurde zum einen die thematische Vielfalt des Fachgebiets deutlich, zum anderen zeigte sich, dass wir in Deutschland insgesamt gut vertreten sind, es sich aber auch lohnt, Schnittmengen mit anderen Fachgebieten wie zum Beispiel der Mikrobiologie, Gynäkologie oder Psychiatrie verstärkt auszuloten. Verfangen hat sich bei mir eine Bemerkung aus dem Grußwort des neuen ersten Vorsitzenden Gerd Burchard als er auf eine (Zitat: „Achtung: Pathos!“) einzigartige Besonderheit der DTG hinwies, die darin bestünde, dass sie die einzige Fachgesellschaft sei, die sich ganz wesentlich mit gesundheitlichen Problemen von Menschen anderswo und nicht nur in Deutschland selbst auseinandersetze. Natürlich sind Tropen-, Reise-, Migrantenmedizin eng verwoben, was durch den Zusatz ,internationale Gesundheit' im Namen unserer Gesellschaft ja auch zum Ausdruck kommen soll. Aber es bietet sich dabei unserer Fachgesellschaft die Chance, die gleichsam auch als Auftrag aufgefasst werden kann, sich auch Themen wie ,emerging infections', ,migrating pathogens', Antibiotikaresistenz sowie zum Beispiel der Bedeutung der heutigen Tierzucht auf die vorgenannten Themen mit einer über unsere Landesgrenze hinaus reichenden Perspektive anzunehmen. Deutlich wurde auch, dass sich das Fachgebiet Tropenmedizin/Medizin in den Tropen verstärkt auch nicht übertragbaren Krankheiten widmet. Inwieweit es uns aber als vorwiegend infektiologisch/epidemiologisch geprägten Ärzten und Wissenschaftlern gelingen kann, sich hierzu auch konkret der Verbesserung des klinischen Patientenmanagements vor Ort anzunehmen beziehungsweise Expertise aus anderen Fachgebieten wie der Kardiologie oder der Endokrinologie einzubinden, wird sich zeigen. Sinnvoll erscheint es in jedem Fall, über einen verstärkten Wissenstransfer jenseits der Infektiologie/Epidemiologie Aktivitäten vor Ort in ressourcenschwachen Ländern selbst zu fördern.

Der Schwerpunkt dieser Ausgabe der FTR liegt - neben einem Aufsatz zur Differenzialdiagnose Eosinophilie - im Wesentlichen auf der Luft- und Raumfahrtmedizin. Petra Frings-Meuthen und Jörn Rittweger zeigen in ihrem Aufsatz zum Knochenstoffwechsel in der Schwerelosigkeit interessante Zusammenhänge von zum Beispiel Immobilisierung und übermäßiger Kochsalzzufuhr mit der Knochendichte auf. Dass sich diese in der Schwerelosigkeit beziehungsweise experimentellen Raumfahrtmedizin gewonnenen Erkenntnisse auch konkret in prophylaktische und therapeutische Ansätze auf Erden umsetzen lassen, greift Günter Ruyters in seinem Beitrag „Schwerelos für Deutschland“ auf. Gleich 2 Themenarbeiten beziehen sich auf das Flugzeug: die Kasuistik zur Schallleitungsschwerhörigkeit bei einem Jagdbomberpiloten von Andreas Knöffler sowie das für alle Reisemediziner sehr relevante Thema zur Flugreisetauglichkeit von vorerkrankten Patienten. Jörg Siedenburg hat sich dabei viel Mühe gemacht, verschiedene chronische Erkrankungen wie Herz- und Lungenkrankheiten oder Diabetes in Bezug zur Flugtauglichkeit einzuordnen und bereichert seinen Artikel mit einer Reihe informativer Tabellen. 